\title{
OPERATORS AND HARMONIC ANALYSIS ON THE SPHERE $\left({ }^{1}\right)$
}

BY

\author{
CHARLES F. DUNKL
}

Introduction. The main result of this paper concerns operators which commute with all rotations on certain spaces of functions on $S^{k}$, the $k$-dimensional sphere $\left(k \geqq 2\right.$ ), namely $C, L^{1}, L^{\infty}$. The proofs use harmonic analysis of various spaces of functions and measures on $S^{k}$, which involves the ultraspherical polynomials.

Notation. $S^{k}$ admits a group of rotations, namely the special orthogonal group $\mathrm{SO}_{k+1}$. The result of the action of the rotation $\alpha$ on the point $x$ will be denoted by $x \alpha$. The "rotation" operator $R_{\alpha}$ acting on functions $(f)$ and measures $(\mu)$ is defined by

$$
\begin{aligned}
& R_{\alpha} f(x)=f(x \alpha) \text { for all } x \in S^{k} \\
& R_{\alpha} \mu(E)=\mu(E \alpha) \text { for all } \mu \text {-measurable subsets } E \text { of } S^{k}
\end{aligned}
$$

$P_{n}^{\lambda}$ is the ultraspherical polynomial of index $\lambda$ and degree $n$ (normalized by $\left.P_{n}^{\lambda}(1)=1\right)$. Considering $S^{k}$ imbedded as the unit sphere in $\mathbf{R}^{k+1}$, let $x \cdot y$ be the ordinary inner product of the vectors which correspond to the points $x$ and $y$ $(-1 \leqq x \cdot y \leqq 1) . S^{k}$ has a unique rotation invariant Borel measure, say $m$, such that $m\left(S^{k}\right)=1$, and the use of this measure is implied by notations such as " $d x$ "; further let $L^{p}\left(S^{k}\right)=L^{p}\left(S^{k}, m\right)$.

Statement of results. With each $\mu \in M\left(S^{k}\right)$, the space of finite regular Borel measures on $S^{k}$, and each $f \in L^{1}\left(S^{k}\right)$, the following continuous functions are associated:

$$
\tilde{\mu}_{n}(x)=\int_{S^{k}} P_{n}^{(k-1) / 2}(x \cdot y) d \mu(y) \quad n=0,1,2, \ldots
$$

and

$$
\tilde{f}_{n}(x)=\int_{S^{k}} P_{n}^{(k-1) / 2}(x \cdot y) f(y) d y \quad n=0,1,2, \ldots
$$

respectively. The sequence $\left\{\tilde{\mu}_{n}\right\}\left[\left\{\tilde{f}_{n}\right\}\right]$ determines $\mu[f]$ uniquely. We choose a north

Received by the editors January 26, 1966.

(1) This paper is a portion of the author's doctoral thesis, which was written under the direction of Professor Walter Rudin at the University of Wisconsin, Madison. The writing of this paper was supported in part by the NSF project "Applications of Functional Analysis" at Princeton University. 
pole $p$ on $S^{k}$ and then define the space of zonal measures to be the set of all $\mu \in M\left(S^{k}\right)$ which are invariant under all rotations which leave $p$ fixed (i.e., $R_{\alpha} \mu=\mu$ for all $\alpha$ such that $p \alpha=p$ ). The zonal functions are defined to be the integrable functions on $S^{k}$ satisfying the same invariance condition. If $\mu$ is a zonal measure then it is determined by a complex sequence $\left\{\hat{\mu}_{n}: n=0,1,2, \ldots\right\}$ where $\hat{\mu}_{n}=\tilde{\mu}_{n}(p)$ and $\tilde{\mu}_{n}(x)=\hat{\mu}_{n} P_{n}^{(k-1) / 2}(p \cdot x)$ for $n=0,1,2, \ldots$ (similarly for zonal functions). The main theorems $(\S \S 8,10)$ state that to each bounded endomorphism $T$ of $C\left(S^{k}\right)$ or of $L^{1}\left(S^{k}\right)$ which commutes with all rotations (i.e., all $R_{\alpha}$ ) there corresponds a zonal measure $\mu$ such that

$$
(T f)_{n}(x)=\hat{\mu}_{n} \tilde{f}_{n}(x) \quad \text { for } n=0,1,2, \ldots
$$

Another result ( $\S 6)$ is that for any infinite subset $E$ of the nonnegative integers the set of restrictions to $E$ of all the sequences $\left\{\hat{f}_{n}\right\}$, where $f$ is a zonal function, is always a proper subset of the space of all complex functions on $E$ which tend to zero at infinity.

\section{Harmonic Analysis}

1. Introduction to the convolution. The chief tool in this work is the convolution operation which may be defined between a zonal measure (function) and an ordinary measure (function) on $S^{k}$. The ultraspherical polynomials of index $(k-1) / 2$ are closely connected with this operation and make possible the determination of the complex homomorphisms of resulting Banach algebras. Specifically the following are Banach algebras under addition and convolution: the spaces of the zonal measures, the zonal $L^{q}$-functions for some $q, 1 \leqq q \leqq \infty$, and the zonal continuous functions.

We write $G$ for $\mathrm{SO}_{k+1}$ and $S$ for $S^{k}$. For any $x \in S$ let $G_{x}$ be the (closed) subgroup of $G$ leaving $x$ fixed. There is a homeomorphism between the orbit space $S / G_{p}$ and the closed interval $[-1,1]$ induced by the continuous map $x \rightarrow p \cdot x$, that is, if $\alpha \in G_{p}$ then $p \cdot x=p \cdot x \alpha$, and if $p \cdot x=p \cdot y$ then $y=x \alpha$ for some $\alpha \in G_{p}$. (The proofs for this statement as well as for the other statements of this section are routine and can be found in [3].) Thus there is an isomorphism between zonal functions and functions on $[-1,1]$. Under this isomorphism the space of zonal functions maps isometrically onto $L^{1}\left([-1,1], \Omega_{(k-1) / 2}\right)$ where $\Omega_{\lambda}$ is the measure $a_{\lambda}\left(1-t^{2}\right)^{\lambda-1 / 2} d t$ ( $a_{\lambda}$ is such that $\left.\int_{-1}^{1} d \Omega_{\lambda}(t)=1\right)$. The polynomials $P_{n}^{\lambda}(t), n=0,1,2, \ldots$, form a complete orthogonal set on $[-1,1]$ with respect to the measure $\Omega_{\lambda}$. Hence the functions [of $x \in S$ ] $P_{n}^{(k-1) / 2}(p \cdot x)$ form a complete orthogonal set of zonal functions.

\section{Definitions and properties of convolution.}

Definition. Let $x \in S$ and define $C(S ; x)=\left\{f \in C(S): R_{\alpha} f=f\right.$ for all $\left.\alpha \in G_{x}\right\}$ and similarly $L^{q}(S ; x)$ and $M(S ; x)$. 
In particular for $x=p$ the above spaces are the zonal ones. Each of the above is a Banach space under the usual norm. For any $x \in S$ we define the map $\varphi_{x}$ from $C(S ; p), L^{q}(S ; p), M(S ; p)$ to $C(S ; x), L^{q}(S ; x), M(S ; x)$ respectively, by using the following fact: if $\alpha, \beta \in G$ such that $x \alpha=x \beta=p$, and $\mu \in M(S ; p)$ then $R_{\alpha} \mu=$ $R_{\beta} \mu \in M(S ; x)$; so we define $\varphi_{x} \mu$ to be $R_{\alpha} \mu$. It is easy to see that $\varphi_{x}$ is an isometric isomorphism onto.

Next we relate the zonal functions to functions on $[-1,1]$. Let $I_{p}$ be the map $x \rightarrow p \cdot x$ of $S$ onto $[-1,1]$. If $f$ is a zonal function, then by a previous remark $f$ is constant on each set $I_{p}^{-1}(t), t \in[-1,1]$, and hence there is a function on $[-1,1]$, which we denote by $\psi_{p} f$, such that $\psi_{p} f(t)=f\left(I_{p}^{-1}(t)\right) . \psi_{p}$ is a $1-1$ map. By an application of the Riesz representation theorem it is easily shown that the dual space of $C(S ; p)$ is $M(S ; p)$, and we use this fact to give an implicit definition of the convolution of zonal measures.

Definition. Suppose $\mu, \nu \in M(S ; p)$ then the map $f \rightarrow \int_{S} \int_{S} \psi_{p} f(x \cdot y) d \mu(x) d \nu(y)$ for $f \in C(S ; p)$ defines a bounded linear functional on $C(S ; p)$ and thus there exists a unique zonal measure $\mu * \nu$ such that

$$
\int_{S} f d(\mu * \nu)=\int_{S} \int_{S} \psi_{p} f(x \cdot y) d \mu(x) d \nu(y)
$$

This convolution is commutative, distributive with addition; $\|\mu * \nu\| \leqq\|\mu\|\|\nu\|$ and $\mu, \nu \geqq 0$ implies $\mu * \nu \geqq 0$ and $\|\mu * \nu\|=\|\mu\|\|\nu\|$. There is an obvious imbedding of $L^{1}(S ; p)$ in $M(S ; p)$, namely $f \rightarrow m_{f}$, where $d m_{f}(x)=f(x) d x$. Thus convolution is defined in $L^{1}(S ; p)$, and gives absolutely continuous $(\ll m)$ measures which may be taken as elements of $L^{1}(S ; p)$, as the following results show:

THEOREM 2a. Suppose $f \in L^{q}(S), 1 \leqq q \leqq \infty,[C(S)]$ and $\mu \in M(S ; p)$ then the formula

$$
f * \mu(x)=\int_{S} f(y) d \varphi_{x} \mu(y)
$$

defines an element of $L^{q}(S)[C(S)]$ with $\|f * \mu\|_{q} \leqq\|f\|_{q}\|\mu\|$. Further if $f$ is zonal then so is $f * \mu$, and $m_{(f * \mu)}=m_{f} * \mu$. $\left(m_{f} * \mu\right.$ is defined above. $)$

Corollary 2b. Suppose $f \in L^{1}(S), g, h \in L^{1}(S ; p)$ then

(i) $g * h(x)=\int_{S} g(y) \varphi_{x} h(y) d y=\int_{S} \psi_{p} g(p \cdot y) \psi_{p} h(y \cdot x) d y$,

(ii) $\psi_{p}(g * h)\left(x_{1} \cdot x_{2}\right)=\int_{S} \psi_{p} g\left(x_{1} \cdot y\right) \psi_{p} h\left(y \cdot x_{2}\right) d y$,

(iii) $f * g(x)=\int_{S} f(y) \varphi_{x} g(y) d y=\int_{S} f(y) \psi_{p} g(x \cdot y) d y$,

(iv) $R_{\alpha}(f * g)=\left(R_{\alpha} f\right) * g$ for all $\alpha \in G$.

Further, convolution can be shown to be associative; in general, if $f \in L^{1}(S)$, $\mu, \nu \in M(S ; p)$ then $f *(\mu * \nu)=(f * \mu) * \nu$. Thus $M(S ; p)$ is a Banach algebra and $L^{1}(S ; p)$ is a closed ideal in it. 
3. Related results. The convolution used here is a special case (at least for the $L^{1}$ and $C$ cases) of the mutually independent results of Bochner [1] and Gel'fand [5]. Bochner discovered a convolution structure for $L^{1}\left([-1,1], \Omega_{\lambda}\right)$, for each $\lambda>0$. Recall from $\S 1$ that $d \Omega_{\lambda}(t)=a_{\lambda}\left(1-t^{2}\right)^{\lambda-1 / 2} d t$. The resulting Banach algebra has its complex homomorphisms given by $f \rightarrow \int_{-1}^{1} f(t) P_{n}^{\lambda}(t) d \Omega_{\lambda}(t) \quad n=0,1,2, \ldots$ (Hirschman [8]). As mentioned above, the space of zonal functions on $S^{k}$ is isomorphic to $L^{1}\left([-1,1], \Omega_{(k-1) / 2}\right)$ and the "spherical" convolution is isomorphic to Bochner's (for the case $\lambda=(k-1) / 2$ ).

Gel'fand's work concerns homogeneous spaces, and is discussed in Helgason's book [6], to which the reader is referred for definitions. Briefly, let $(G, K)$ be a Riemannian symmetric pair (implying that $G$ is a connected Lie group, $K$ is a compact subgroup, and there exists an involutive analytic automorphism of $G$ leaving $K$ fixed), then the set of all continuous functions with compact support on $G$ which are bi-invariant under $K$ forms a commutative algebra under addition and the ordinary locally-compact group-theoretic convolution. In particular $S^{k} \cong$ $\mathrm{SO}_{k+1} / \mathrm{SO}_{k}$ (i.e., $S \cong G / G_{p}$ ) so $S$ is a homogeneous space, the space of right cosets of $G_{p}, p$ corresponding to $G_{p}$. The functions on $G$ which are left-invariant under $G_{p}$ $\left(f(\alpha)=f(\beta \alpha)\right.$ for all $\left.\beta \in G_{p}\right)$ correspond to functions on $S$, while the bi-invariant functions on $G$ correspond to zonal functions. It is not hard to show that convolution on $G$ when restricted to the bi-invariant functions gives rise to the same operation on zonal functions as the "spherical" convolution.

4. Harmonic analysis of $L^{1}(S), L^{1}(S ; p)$ and $M(S ; p)$. We now need the ultraspherical polynomials, and they are defined by the relation

$$
\left(1-2 r t+r^{2}\right)^{-\lambda}=\sum_{n=0}^{\infty} \frac{\Gamma(2 \lambda+n)}{n ! \Gamma(2 \lambda)} P_{n}^{\lambda}(t) r^{n}
$$

Then $P_{n}^{\lambda}(1)=1$ and $\left|P_{n}^{\lambda}(t)\right| \leqq 1$ for $-1 \leqq t \leqq 1$. Henceforth we set $\lambda=(k-1) / 2$; then (Erdélyi [4]) the set $\left\{\psi_{p}^{-1} P_{n}^{\lambda}: n=0,1,2, \ldots\right\}$ forms a complete orthogonal set in $L^{2}(S ; p)$. [Note: $\psi_{p}^{-1} P_{n}^{\lambda}(x) \equiv P_{n}^{\lambda}(p \cdot x)$.] Suppose $\mu \in M(S ; p), n=0,1,2, \ldots$, then we define the $n$th Gegenbauer coefficient $\hat{\mu}_{n}$ of $\mu$ by $\hat{\mu}_{n}=\int_{S} P_{n}^{\lambda}(p \cdot x) d \mu(x)$, and for $f \in L^{1}(S ; p)$ define $\hat{f}_{n}=\int_{S} P_{n}^{\lambda}(p \cdot x) f(x) d x$. Note $\left|\hat{\mu}_{n}\right| \leqq\|\mu\|$ and $\left|\hat{f}_{n}\right| \leqq\|f\|_{1}$.

By using the theory of spherical functions on homogeneous spaces [6] and E. Cartan's [2] result that the $\left\{\psi_{p}^{-1} P_{n}^{\lambda}: n=0,1,2, \ldots\right\}$ are the spherical functions on $S$ we obtain the formula:

$$
\int_{G_{p}} P_{n}^{\lambda}(x \cdot y \alpha) d \alpha=P_{n}^{\lambda}(x \cdot p) P_{n}^{\lambda}(p \cdot y)
$$

for all $x, y \in S$, where $d \alpha$ is the normalized Haar measure of $G_{p}$.

In turn this formula leads to the following: for $\mu, \nu \in M(S ; p)$

$$
(\mu * \nu)_{n}^{\wedge}=\hat{\mu}_{n} \hat{\nu}_{n} \quad n=0,1,2, \ldots,
$$


so that the maps $\mu \rightarrow \hat{\mu}_{n}$ are complex homomorphisms of $M(S ; p)$. Hirschman's work contains the fact that the maps $f \rightarrow \hat{f}_{n}\left(f \in L^{1}(S ; p)\right) n=0,1,2, \ldots$ are exactly the nontrivial complex homomorphisms of $L^{1}(S ; p)$, thus the maximal ideal space of $L^{1}(S ; p)$ is homeomorphic to the space of nonnegative integers, since the Gel'fand topology is obviously the discrete one.

We note that $\left\{\psi_{p}^{-1} P_{n}^{\lambda}: n=0,1,2, \ldots\right\}$ is dense in $C(S ; p)$ hence $\hat{\mu}_{n}=0$ for all $n$ implies $\mu=0$.

For functions in $L^{1}(S)$ we use the expansion in terms of components with respect to the class of subspaces where each subspace consists of the restrictions to $S^{k}$ (the unit sphere in $\mathbf{R}^{k+1}$ ) of all the harmonic homogeneous polynomials in $\mathbf{R}^{k+1}$ of fixed degree. The sum of these subspaces is dense in $L^{1}(S)$. For $f \in L^{1}(S)$, $n=0,1,2, \ldots$, we define $\tilde{f}_{n}(x)=f * \psi_{p}^{-1} P_{n}^{\lambda}(x)=\int_{S} f(y) P_{n}^{\lambda}(x \cdot y) d y$. Given $\left\{\tilde{f}_{n}\right\}, f$ can be reconstructed, in fact

$$
f=\sum_{n=0}^{\infty}\left(\begin{array}{c}
n+k-2 \\
n
\end{array}\right)\left(\frac{2 n}{k-1}+1\right) f_{n}
$$

where this series is summable, at least in the $(C, k)$ sense, in the norm (Koschmieder [9]).

4a. The main relations between convolution and the above expansion are as follows: Let $f \in L^{1}(S), g \in L^{1}(S ; p), \mu \in M(S ; p), n=0,1,2, \ldots$ then

(i) $(f * g)_{n}^{\tilde{n}}(x)=\hat{g}_{n} \tilde{f}_{n}(x)$,

(ii) $(f * \mu)_{n}^{\tilde{n}}(x)=\hat{\mu}_{n} \tilde{f}_{n}(x)$,

(iii) $\tilde{g}_{n}(x)=\hat{g}_{n} \psi_{p}^{-1} P_{n}^{\lambda}(x)=\hat{g}_{n} P_{n}^{\lambda}(p \cdot x)$,

$$
g=\sum_{n=0}^{\infty}\left(\begin{array}{c}
n+k-2 \\
n
\end{array}\right)\left(\frac{2 n}{k-1}+1\right) \hat{g}_{n} \psi_{p}^{-1} P_{n}^{\lambda} \text { summation as above. }
$$

4b. There exists a class $\left\{u_{v}\right\}$ of zonal functions, one for each neighborhood $V$ of 1 in $[-1,1]$ which is an approximate identity for $C(S ; p)$ and $L^{1}(S ; p)$, that is, $u_{v} \geqq 0, u_{v}=0$ off $V, \int_{S} u_{v}(x) d x=1$, and if $f \in C(S)\left[L^{1}(S)\right]$ then $\left\|f-f * u_{v}\right\| \rightarrow 0$ as $V$ contracts to 1 (using the appropriate norm).

5. Measures. A closer examination of the geometric effect of convolution shows that the convolution of two continuous (zero on any countable set) measures is absolutely continuous and this leads to the determination of all idempotent zonal measures and of the maximal ideal space of $M(S ; p)$. Note that $M(S ; p)$ has an algebraic identity, $\delta$, the unit mass at $p$ (i.e., $\delta E=1$ if $p \in E, 0$ if $p \notin E$ ). Let $\tilde{p}$ be the antipodal point of $p$ on $S\left(\tilde{p}=-p\right.$ in the $\mathbf{R}^{k+1}$-sense $)$ and let $M_{c}(S ; p)=$ $\{\mu \in M(S ; p):|\mu|\{p, \tilde{p}\}=0\}$. This is exactly the set of continuous zonal measures since the only countable set invariant under $G_{p}$ is $\{p, \tilde{p}\}$.

THEOREM 5a. Suppose $\mu, \nu \in M_{c}(S ; p)$, then $\mu * \nu \ll m$. 
Outline of the proof.

(i) If $\mu \in M(S ; p)$ has the property that $\mu E=0$ whenever $E$ is a Borel set $\subset S$ such that $E \alpha=E$ for all $\alpha \in G_{p}$ (i.e., $\chi_{E} \in L^{1}(S ; p)$ ) and $m E=0$, then $\mu \ll m$.

(ii) For such sets $E, \psi_{p} \chi_{E}(t)=0$ a.e. for $-1 \leqq t \leqq 1$. The above statements are straightforward to prove.

(iii) For a Borel set $E$, such that $\chi_{E} \in L^{1}(S ; p)$ and $\mu, \nu \in M(S ; p)$

$$
\mu * \nu(E)=\int_{S} d \mu(x) \int_{S} d \nu(y) \int_{G_{p}} \psi_{p} \chi_{E}(x \alpha \cdot y) d \alpha .
$$

This is derived as follows: For $f \in C(S), \mu \in M(S ; p)$

$$
\int_{S} f d \mu=\int_{S} d \mu(x) \int_{G_{p}} f(x \alpha) d \alpha
$$

because

$$
\int_{S}\left(R_{\alpha} f\right) d \mu=\int_{S} f d\left(R_{\alpha^{-1}} \mu\right)=\int_{S} f d \mu
$$

for all $\alpha \in G_{p}$ and hence

$$
\int_{S} f d \mu=\int_{G_{p}} d \alpha \int_{S} f(x \alpha) d \mu(x)=\int_{S} d \mu(x) \int_{G_{p}} f(x \alpha) d \alpha .
$$

Further if $f \in C(S ; p)$ then

$$
\begin{aligned}
\int_{S} f d(\mu * \nu) & =\int_{S} \int_{S} \psi_{p} f(x \cdot y) d \mu(x) d \nu(y) \\
& =\int_{S} d \nu(y) \int_{S} \psi_{p} f(x \cdot y) d \mu(x) \\
& =\int_{S} d \nu(y) \int_{S} d \mu(x) \int_{G_{p}} \psi_{p} f(x \alpha \cdot y) d \alpha
\end{aligned}
$$

since $\psi_{p} f(x \cdot y)$ is continuous in $x$ for a fixed $y$. The formula is then extended to Borel functions.

(iv) $\int_{G_{p}} \psi_{p} \chi_{E}(x \alpha \cdot y) d \alpha=c \int_{-1}^{1} \psi_{p} \chi_{E}\left(s t+\left[\left(1-s^{2}\right)\left(1-t^{2}\right)\right]^{1 / 2} u\right)\left(1-u^{2}\right)^{(k-3) / 2} d u$

where $s=p \cdot x, t=p \cdot y$ and $c$ is a constant depending only on $k$. This formula is derived by changing the integral over $G_{p}$ to an integral over $S_{x}^{k-1}=\{z \in S: p \cdot z=p \cdot x\}$, a $(k-1)$-sphere, and using a spherical polar coordinate system for $S_{x}^{k-1}$.

(v) The above integral vanishes if $s, t \neq 1,-1$ and $\psi_{p} \chi_{E}=0$ a.e.

(vi) If $\mu, \nu \in M_{c}(S ; p)$ and $E$ as in (i) then $\mu * \nu(E)=0$ since $s, t=1$ or -1 only for $x, y=p$ or $\tilde{p}$ where both $\mu$ and $\nu$ have no mass.

By (vi) and (i) $\mu * \nu \ll m$. 
TheOREM 5b. $M_{c}(S ; p)$ is an ideal in $M(S ; p)$ and consists exactly of those $\mu$ satisfying $\hat{\mu}_{n}=o(1)$ as $n \rightarrow \infty$.

Proof. (i) Let $\mu \in M_{c}(S ; p)$, then $\mu^{2}(=\mu * \mu) \in L^{1}(S ; p)$ and $\left(\hat{\mu}_{n}\right)^{2}=\left(\mu^{2}\right)_{n}^{\wedge} \rightarrow 0$ as $n \rightarrow \infty$ (Gel'fand representation theorem for $\left.L^{1}(S ; p)\right)$, hence $\hat{\mu}_{n}=o(1)$ as $n \rightarrow \infty$.

(ii) Let $\mu \in M(S ; p)$ and suppose $\hat{\mu}_{n}=o(1)$ as $n \rightarrow \infty$. Let $\delta^{\prime}$ be the unit mass at $\tilde{p}$, then every $\mu \in M(S ; p)$ has a unique representation as $\mu=a \delta+b \delta^{\prime}+\mu_{c}$ where $a, b \in \mathbf{C}$ and $\mu_{c} \in M_{c}(S ; p)$. Note that

$$
\left(\delta^{\prime}\right)_{n}^{\wedge}=\int_{S}\left(\psi_{p}^{-1} P_{n}^{\lambda}\right) d \delta^{\prime}=\psi_{p}^{-1} P_{n}^{\lambda}(\tilde{p})=P_{n}^{\lambda}(-1)=(-1)^{n}
$$

so $\hat{\mu}_{n}=a+b(-1)^{n}+\left(\mu_{c}\right)_{n}$. As $n \rightarrow \infty, \hat{\mu}_{n} \rightarrow 0$ and $\left(\mu_{c}\right)_{n} \rightarrow 0$; hence $a=b=0$ and $\mu \in M_{c}(S ; p)$. Q.E.D.

THEOREM 5c. The set of nontrivial complex homomorphisms of $M(S ; p)$ consists of the set $\left\{H_{n}: n=0,1,2, \ldots\right\}\left[H_{n}(\mu)=\hat{\mu}_{n}\right]$ and two other maps $H_{E}$ and $H_{O}$ which both vanish on $M_{c}(S ; p)$ and are given by: $H_{E}(\mu)=\mu\{p\}+\mu\{\tilde{p}\}, H_{O}(\mu)=\mu\{p\}-\mu\{\tilde{p}\}$. ( $E$ and $O$ stand for even and odd, respectively.) Further the maximal ideal space is compact in the Gel'fand (weak) topology, and $\lim _{n \rightarrow \infty} H_{2 n}=H_{E}, \lim _{n \rightarrow \infty} H_{2 n+1}=H_{O}$. All the points $H_{n}$ are isolated.

Proof. Let $H$ be a nontrivial complex homomorphism of $M(S ; p)$, then either (i) $H(\nu) \neq 0$ for some $\nu \in M_{c}(S ; p)$, or (ii) $H\left(M_{c}\right)=0$.

Case (i): Since $L^{1}(S ; p)$ is a subalgebra of $M(S ; p)$ and $g=\nu * \nu \in L^{1}(S ; p)$ we have $H(g) \neq 0$ and thus $H$ is nontrivial on $L^{1}(S ; p)$. It is known [8] that $\left\{H_{n}: n=0,1,2, \ldots\right\}$ is the set of all complex homomorphisms of $L^{1}(S ; p)$ hence there is an integer $n \geqq 0$ such that $H(f)=\hat{f}_{n}$ for all $f \in L^{1}(S ; p) . L^{1}(S ; p)$ is an ideal in $M(S ; p)$ so that for each $\mu \in M(S ; p), \mu * g \in L^{1}(S ; p)$ and

$$
H(\mu)=H(\mu * g) / H(g)=(\mu * g)_{n} / \hat{g}_{n}=\hat{\mu}_{n}
$$

so $H=H_{n}$.

Case (ii): Represent $\mu \in M(S ; p)$ as $\mu=a \delta+b \delta^{\prime}+\mu_{c}$ where $a, b \in \mathbf{C}, \mu_{c} \in M_{c}(S ; p)$. Then, by hypothesis, $H(\mu)=a H(\delta)+b H\left(\delta^{\prime}\right)$. Since $\delta$ is the identity, $H(\delta)=1$, and since $\delta^{\prime} * \delta^{\prime}=\delta, H\left(\delta^{\prime}\right)= \pm 1$. To prove $\delta^{\prime} * \delta^{\prime}=\delta$ we observe that for any $f \in C(S ; p)$

$$
\begin{aligned}
\int_{S} f d\left(\delta^{\prime} * \delta^{\prime}\right) & =\int_{S} \int_{S} \psi_{p} f(x \cdot y) d \delta^{\prime}(x) d \delta^{\prime}(y) \\
& =\psi_{p} f(1)=f(p)=\int_{S} f d \delta
\end{aligned}
$$


Let $H_{E}$ be defined by $H_{E}(\mu)=a+b$ (i.e., $\left.H_{E}\left(\delta^{\prime}\right)=1\right)$ and $H_{O}$ as $H_{O}(\mu)=a-b$ (i.e., $\left.H_{o}\left(\delta^{\prime}\right)=-1\right)$. For each $\mu \in M(S ; p)$,

$$
H_{2 n}(\mu)=a H_{2 n}(\delta)+b H_{2 n}\left(\delta^{\prime}\right)+H_{2 n}\left(\mu_{c}\right)=a+b+\left(\mu_{c}\right) \hat{2 n} \rightarrow a+b=H_{E}(\mu)
$$

as $n \rightarrow \infty$ and $H_{2 n+1}(\mu)=a-b+\left(\mu_{c}\right)_{2 n+1} \rightarrow a-b=H_{O}(\mu)$ as $n \rightarrow \infty$, hence $H_{E}$ and $H_{O}$ are complex homomorphisms.

\section{Idempotent measures.}

THEOREM 5d. The set of idempotent zonal measures is in 1-1 correspondence with the set of all continuous functions from the maximal ideal space of $M(S ; p)$ into $\{0,1\}$.

Proof. For $\mu \in M(S ; p)$, let $\hat{\mu}$ denote the Gel'fand transform of $\mu$, that is, $\hat{\mu}(H)=H(\mu)$ for each $H$ in the maximal ideal space, then $\hat{\mu}$ is a continuous function. If further $\mu * \mu=\mu$ then $(\hat{\mu})^{2}=\hat{\mu}$, so $\hat{\mu}$ takes its values in $\{0,1\}$.

Conversely let $\varphi$ be a continuous function from the maximal ideal space into $\{0,1\}$, then since $\lim _{n \rightarrow \infty} \varphi\left(H_{2 n}\right)=\varphi\left(H_{E}\right), \lim _{n \rightarrow \infty} \varphi\left(H_{2 n+1}\right)=\varphi\left(H_{O}\right)$ and $\{0,1\}$ is discrete, it follows that $\varphi\left(H_{2 n}\right)=\varphi\left(H_{E}\right)$ and $\varphi\left(H_{2 n+1}\right)=\varphi\left(H_{O}\right)$ for all but a finite number of integers $n$. There are four cases for the pair $\left(\varphi\left(H_{E}\right), \varphi\left(H_{O}\right)\right)$, namely (1) $(1,1),(2)(1,0),(3)(0,1)$, (4) $(0,0)$. Define the measures $\mu_{1}=\delta, \mu_{2}=\frac{1}{2} \delta+\frac{1}{2} \delta^{\prime}$, $\mu_{3}=\frac{1}{2} \delta-\frac{1}{2} \delta^{\prime}, \quad \mu_{4}=0$, then $\left(\mu_{j}\right)^{\wedge}\left(H_{2 n}\right)=\left(\mu_{j}\right)^{\wedge}\left(H_{E}\right)$ and $\left(\mu_{j}\right)^{\wedge}\left(H_{2 n+1}\right)=\left(\mu_{j}\right)^{\wedge}\left(H_{O}\right)$ for all $n, j=1,2,3,4$. Suppose $\varphi$ is in case $j$, then $\varphi-\left(\mu_{j}\right)^{\wedge}$ is zero for all but a finite number of points $H_{n}$. There is a continuous zonal measure $\lambda$ (corresponding to a polynomial) such that $\varphi-\left(\mu_{j}\right)^{\wedge}=\hat{\lambda}$ everywhere, hence $\varphi=\left(\mu_{j}\right)^{\wedge}+\hat{\lambda}$. Let $\mu=\mu_{j}+\lambda$, then $\hat{\mu}=\varphi$ and $\mu * \mu=\mu$. Q.E.D.

REMARK. The theorem can be stated as follows: To any sequence $\left\{c_{n}\right\} \subset\{0,1\}$ which is of period 2 for all but a finite number of places there exists an idempotent zonal measure $\mu$, such that $\hat{\mu}_{n}=c_{n}$ for all $n$, and this exhausts all idempotent zonal measures.

6. Sidon sets. Let $E \subset\{0,1,2, \ldots\}$ then $C(E)$ denotes the space of all bounded functions on $E$, and $C_{0}(E)$ denotes the space of all (bounded) functions on $E$ tending to zero at infinity. We say $E$ is a Sidon set for $L^{1}(S ; p)$ if for every $\varphi \in C_{0}(E)$ there corresponds a zonal function $f$ such that $\hat{f}_{n}=\varphi(n)$ for all $n \in E$. (This definition is motivated by the concept of a Sidon set for a compact abelian group [10, p. 121].)

THEOREM 6. The following statements are equivalent:

(a) $E$ is a Sidon set.

(b) To every $\varphi \in C(E)$ there corresponds a zonal measure $\mu$ (i.e., $\mu \in M(S ; p)$ ) such that $\hat{\mu}_{n}=\varphi(n)$ for all $n \in E$.

(c) $E$ is finite. 
Proof. (a) implies (b): Assume (a), then for every $\varphi \in C_{0}(E)$ there exists an $f \in L^{1}(S ; p)$ such that $\hat{f}_{n}=\varphi(n)$ for all $n \in E$ and $\|\varphi\|_{\infty} \leqq\|\hat{f}\|_{\infty} \leqq\|f\|_{1}$. Let $L^{\prime}=$ $\left\{f \in L^{1}(S ; p): \hat{f}_{n}=0\right.$ for all $\left.n \in E\right\}$, which is a closed subspace of $L^{1}(S ; p)$. Then by hypothesis $L^{1}(S ; p) / L^{\prime}$ is isomorphic to $C_{0}(E)$ and by the closed graph theorem the map is continuous. The definition of the quotient norm shows that there exists a constant $B$, depending only on $E$, such that to each $\varphi \in C_{0}(E)$ there corresponds a function $f \in L^{1}(S ; p)$ with $\hat{f}_{n}=\varphi(n)$ for all $n \in E$ and $\|f\|_{1} \leqq B\|\varphi\|_{\infty}$. Let $\sigma \in C(E)$, then there is a sequence $\left\{\varphi_{j}: j=1,2, \ldots\right\} \subset C_{0}(E)$ with $\varphi_{j}$ tending to $\sigma$ pointwise as $j \rightarrow \infty$ and $\left\|\varphi_{j}\right\|_{\infty} \leqq\|\sigma\|_{\infty}$. By the above remarks there is a sequence

$$
\left\{f^{(j)}: j=1,2, \ldots\right\} \subset L^{1}(S ; p)
$$

such that $\left\|f^{(j)}\right\|_{1} \leqq B\left\|\varphi_{j}\right\|_{\infty} \leqq B\|\sigma\|_{\infty}$ and $\left(f^{(j)}\right)_{n}^{\wedge}=\varphi_{j}(n)$ for all $n \in E$. A subsequence of $\left\{f^{(j)}\right\}$ converges $*$-weakly to a measure $\mu \in M(S ; p)$ and hence for each $n \in E$, $\hat{\mu}_{n}=\lim _{j}\left(f^{(j)}\right)_{n}^{\hat{n}}=\lim _{j} \varphi_{j}(n)=\sigma(n)$. Thus (b) holds.

(b) implies (c): We suppose $E$ is infinite and prove it cannot have property (b). $E$ contains an infinite subset of the even integers or of the odd integers, say of the even integers. By $5 \mathrm{c} \lim _{n \rightarrow \infty} \hat{\mu}_{2 n}$ exists for all $\mu \in M(S ; p)$ but then $\lim _{n \rightarrow \infty ; 2 n \in E} \hat{\mu}_{2 n}$ exists and there are functions $\varphi \in C(E)$ such that $\lim _{n \rightarrow \infty ; 2 n \in E} \varphi(2 n)$ does not exist (similarly if $E$ contains an infinite subset of the odd integers).

(c) implies (a): Let

$$
f(x)=\sum_{n \in E}\left(\begin{array}{c}
n+k-2 \\
n
\end{array}\right)\left(\frac{2 n}{k-1}+1\right) \varphi(n) P_{n}^{\lambda}(p \cdot x),
$$

a polynomial. Q.E.D.

\section{Rotation-Commuting Operators on $C(S), L^{1}(S)$ and $L^{\infty}(S)$}

7. Introduction. Suppose that $T$ is a linear transformation defined on some subspace of $L^{1}(S)$, such as $C(S)$ or $L^{\infty}(S)$, with its range a subspace of $L^{1}(S)$, and there is a sequence of complex numbers $\left\{t_{n}: n=0,1,2, \ldots\right\}$ such that $(T f)_{n}^{\tilde{n}}(x)=t_{n} \tilde{f}_{n}(x)$ for all $n$, then we say $T$ is a multiplier transformation. We observe that for all $\alpha \in G,\left(R_{\alpha} f\right)_{n}^{\tilde{n}}=R_{\alpha}\left(\tilde{f}_{n}\right)$, since

$$
\left(R_{\alpha} f\right)_{n}^{\tilde{n}}(x)=\int_{S} R_{\alpha} f(y) P_{n}^{\lambda}(x \cdot y) d y=\int_{S} f(y) P_{n}^{\lambda}\left(x \cdot y \alpha^{-1}\right) d y=\tilde{f}_{n}(x \alpha)=R_{\alpha} \tilde{f}_{n}(x),
$$

for all $f \in L^{1}(S)$, almost all $x \in S$. Thus a multiplier transformation commutes with all rotations, since

$$
\left(R_{\alpha} T f\right)_{n}^{\tilde{n}}(x)=(T f)_{n}^{\tilde{n}}(x \alpha)=t_{n} R_{\alpha} \tilde{f}_{n}(x)=t_{n}\left(R_{\alpha} f\right)_{n}^{\tilde{n}}(x)=\left(T R_{\alpha} f\right)_{\tilde{n}}^{\tilde{n}}(x)
$$

for all $n$, and so $R_{\alpha} T f=T R_{\alpha} f$ (see $\S 4$ ). An important type of multiplier transformation is that of convolution with some fixed element of $M(S ; p)$ or $L^{1}(S ; p)$. 
Let $F$ be a Banach space of functions on $S$, and let $B_{G}(F)$ be the space of bounded linear transformations of $F$ into itself which commute with all rotations. We will show that all elements of $B_{G}\left(L^{1}(S)\right), B_{G}(C(S))$, and those of $B_{G}\left(L^{\infty}(S)\right)$ which are multiplier transformations, arise from convolution with an element of $M(S ; p)$. The map $f \rightarrow T_{\mu} f=f * \mu$ for a fixed $\mu \in M(S ; p)$ is a bounded multiplier transformation for $L^{q}(S), 1 \leqq q \leqq \infty$, and $C(S)$ (see $2 \mathrm{a}$ and $4 \mathrm{a}(\mathrm{ii})$ ).

\section{The case $C(S)$.}

THEOREM 8. There is a Banach algebra isomorphism between $M(S ; p)$ and $B_{G}(C(S))$, given by $\mu \rightarrow T_{\mu}\left(T_{\mu} f=f * \mu\right.$, for all $\left.f \in C(S)\right)$. Thus each element of $B_{G}(C(S))$ is a multiplier transformation.

Proof. From $2 \mathrm{a}$ and $4 \mathrm{a}$ (ii) we have $\left(T_{\mu} f\right)_{n} \tilde{n}(x)=\hat{\mu}_{n} f_{n}(x)$ for all $n$ and $\left\|T_{\mu} f\right\|_{\infty} \leqq$ $\|\mu\|\|f\|_{\infty}$, hence $T_{\mu} \in B_{G}(C(S))$ and $\left\|T_{\mu}\right\| \leqq\|\mu\|$. It will suffice to show that for each $T \in B_{G}(C(S))$ there exists $\mu \in M(S ; p)$ such that $T=T_{\mu}$ and $\|\mu\| \leqq\|T\|$. Let $T \in B_{G}(C(S))$. For each $x \in S$, the map $f \rightarrow T f(x)$ is a bounded linear functional on $C(S)$, hence by the Riesz representation theorem there exists a measure $m_{x} \in M(S)$, with $\left\|m_{x}\right\| \leqq\|T\|$ such that $T f(x)=\int_{S} f(y) d m_{x}(y)$. Since $T \in B_{G}(C(S))$ we must have $T R_{\alpha}=R_{\alpha} T$ for all $\alpha \in G$, so for all $f \in C(S), x \in S$,

$$
\begin{gathered}
T R_{\alpha} f(x)=\int_{S} R_{\alpha} f(y) d m_{x}(y)=\int_{S} f(y) d R_{\alpha}^{-1} m_{x}(y), \\
R_{\alpha} T f(x)=T f(x \alpha)=\int_{S} f(y) d m_{x \alpha}(y),
\end{gathered}
$$

hence $R_{\alpha}^{-1} m_{x}=m_{x \alpha}$. Let $\mu=m_{p}$, then if $\alpha \in G_{p}, R_{\alpha} \mu=m_{p \alpha^{-1}}=m_{p}=\mu$ so $\mu \in M(S ; p)$. Further $m_{p \alpha}=R_{\alpha}^{-1} m_{p}=R_{\alpha}^{-1} \mu=\varphi_{p \alpha} \mu$ for all $\alpha \in G$. Thus

$$
T f(x)=\int_{S} f(y) d \varphi_{x} \mu(y)=f * \mu(x)=T_{\mu} f(x),
$$

and $\|\mu\| \leqq\|T\|$. Combining this with the previous result, we see that the map $\mu \rightarrow T_{\mu}$ is onto and isometric. By the associative law $2 \mathrm{~b}, T_{\mu} T_{\nu}=T_{\nu \bullet \mu}$, and hence $B_{G}(C(S))$ is a commutative Banach algebra. Q.E.D.

9. The case $L^{\infty}(S)$. The main result will follow from several lemmas.

Lemma 9a. Suppose $f \in L^{\infty}(S)$ and has the property that for all $\varepsilon>0$, there is a neighborhood $U$ of the identity in $G$ such that $\left\|f-R_{\alpha} f\right\|_{\infty}<\varepsilon$ for all $\alpha \in U$, then there is a unique $f_{c} \in C(S)$ with $f=f_{c}$ a.e. 
Proof. If $f_{c}$ exists then it is unique. Let $u_{v}$ run through an approximate identity in $L^{1}(S ; p)$ (see $4 \mathrm{~b}$ ). Then the set $\left\{f * u_{v}\right\}$ is bounded by $\|f\|_{\infty}$, and is an equicontinuous family, as the following argument shows: Let $\varepsilon>0$, then there exists a neighborhood $U$ of the identity $G$ such that $\left\|f-R_{\alpha} f\right\|_{\infty}<\varepsilon$ for all $\alpha \in U$. Then

$$
\begin{aligned}
|f * u(x)-f * u(x \alpha)| & =\left|\int_{S} f(y) \psi_{p} u(x \cdot y) d y-\int_{S} f(y) \psi_{p} u(x \alpha \cdot y) d y\right| \\
& =\left|\int_{S}[f(y)-f(y \alpha)] \psi_{p} u(x \cdot y) d y\right| \leqq\left\|f-R_{\alpha} f\right\|_{\infty}\|u\|_{1}<\varepsilon .
\end{aligned}
$$

Thus by Ascoli's theorem $\left\{f * u_{v}\right\}$ contains a subsequence converging to a function $f_{c} \in C(S)$. We show that $\left\|f-f_{c}\right\|_{\infty}=0$ by proving $\left\|f-f * u_{v}\right\|_{\infty} \rightarrow 0$ as $V$ contracts to 1 . For almost all $x$

$$
\left|f(x)-f * u_{v}(x)\right|=\left|\int_{S}[f(x)-f(y)] \psi_{p} u_{v}(x \cdot y) d y\right| \leqq \underset{\{y: x \cdot y \in V\}}{\operatorname{essup}}|f(x)-f(y)| .
$$

Here we need a remark about uniform structures on $S$. Let $U$ and $V$ be neighborhoods of the identity in $G$, and of 1 in $[-1,1]$ respectively, and let

$$
\begin{gathered}
U_{S}=\{(x, y): y=x \alpha \text { some } \alpha \in U\} \quad \text { and } \\
V_{S}=\{(x, y): x \cdot y \in V\} .
\end{gathered}
$$

Then each of the set of all such $U_{S}$ and the set of all such $V_{S}$ is a set of basic entourages for a uniform structure on $S$, which generates the ordinary topology on $S$, thus the two are equivalent, since $S$ is compact.

Now let $U$ be as in the start of the proof, then there is a neighborhood $V$ of 1 in $[-1,1]$ such that $V_{S} \subset U_{S}$, then $\left|f(x)-f * u_{v}(x)\right|<\varepsilon$ for almost all $x$. Q.E.D.

Lemma 9b. Suppose $T \in B_{G}\left(L^{\infty}(S)\right), f \in C(S)$, then there exists $g \in C(S)$ such that $T f=g$ a.e., that is, $T$ maps $C(S)$ into $C(S)$.

Proof. Given $\varepsilon>0$, by the uniform continuity of $f$ there exists a neighborhood $U$ of the identity in $G$ such that $\left\|f-R_{\alpha} f\right\|_{\infty}<\varepsilon /\|T\|$ for all $\alpha \in U$. Then

$$
\left\|T f-R_{\alpha}(T f)\right\|_{\infty}=\left\|T f-T R_{\alpha} f\right\|_{\infty}=\left\|T\left(f-R_{\alpha} f\right)\right\|_{\infty} \leqq\|T\|\left\|f-R_{\alpha} f\right\|_{\infty}<\varepsilon
$$

for all $\alpha \in U$. Thus $T f$ satisfies the hypotheses of Lemma 9a and the proof is finished. Q.E.D.

THEOREM 9c. Suppose $T \in B_{G}\left(L^{\infty}(S)\right)$ and is a multiplier transformation, then $T=T_{\mu}$ for some $\mu \in M(S ; p)$.

Proof. By $9 \mathrm{~b}$ and 8 there exists $\mu \in M(S ; p)$ such that $T f=f * \mu$ for all $f \in C(S)$. Thus $(T f)_{n}^{\tilde{n}}(x)=\hat{\mu}_{n} \tilde{f}_{n}(x)$ for all $n$, for all $f \in C(S)$, but by hypothesis $(T f)_{n}(x)=$ $t_{n} \tilde{f}_{n}(x)$, so $t_{n}=\hat{\mu}_{n}$ for all $n$, and $T=T_{\mu}$. Q.E.D. 
10. The case $L^{1}(S)$. We will investigate the restriction of $T \in B_{G}\left(L^{1}(S)\right)$ to $L^{1}(S ; p)$ and will show that $T$ is a multiplier transformation, which arises from convolution with a measure, by a theorem analogous to one of Helson [7].

THEOREM 10a. Suppose $T \in B_{G}\left(L^{1}(S)\right)$, then $T$ maps $L^{1}(S ; p)$ into itself and is a multiplier transformation on $L^{1}(S ; p)$.

Proof. First we show that $T f \in L^{1}(S ; p)$ if $f \in L^{1}(S ; p)$. Let $\alpha \in G_{p}$ then $R_{\alpha} T f=$ $T R_{\alpha} f=T f$. To show $T$ is a multiplier transformation on $L^{1}(S ; p)$ we need the relation $(T f) * g=f *(T g)$ for all $f, g \in L^{1}(S ; p)$; for then, taking Gegenbauer coefficients, we obtain $(T f)_{n} \hat{g}_{n}=\hat{f}_{n}(T g)_{n}$. Thus $(T f)_{n} / \hat{f}_{n}\left(\hat{f}_{n} \neq 0\right)$ is a constant $t_{n}$ independent of $f$, and hence $(T f)_{n}^{\tilde{n}}(x)=(T f)_{n} \psi_{p}^{-1} P_{n}^{\lambda}(x)=t_{n} \hat{f}_{n} \psi_{p}^{-1} P_{n}^{\lambda}(x)=t_{n} \hat{f}_{n}(x)$. For later use, we actually show that $T(f * g)=f * T g$ for $f \in L^{1}(S), g \in L^{1}(S ; p)$. Following a technique of Rudin [10, p. 74], we take a fixed $b \in L^{\infty}(S)$ and define a bounded linear functional on $L^{1}(S)$ by $f \rightarrow \int_{S}(T f) b d m$. Thus there exists $\beta \in L^{\infty}(S)$ such that $\int_{S}(T f) b d m=\int_{S} f \beta d m$. Let $f \in L^{1}(S), g \in L^{1}(S ; p)$ and note that $T \varphi_{x} g=$ $\varphi_{x} T g$ for all $x \in S$, since $T R_{\alpha}=R_{\alpha} T$ for all $\alpha \in G$, and if $h \in L^{1}(S ; p)$ then

$$
\begin{aligned}
\varphi_{y} h(x)=\psi_{p} h(x \cdot y)=\varphi_{x} h(y) \\
\int_{S}(f * T g) b d m=\int_{S} b(x) d x \int_{S} f(y) \varphi_{x} T g(y) d y \\
=\int_{S} b(x) d x \int_{S} f(y) \varphi_{y} T g(x) d y \\
=\int_{S} f(y) d y \int_{S} b(x) \varphi_{y} T g(x) d x \quad \text { (Fubini's theorem) } \\
=\int_{S} f(y) d y \int_{S} \beta(x) \varphi_{y} g(x) d x \\
=\int_{S} \beta(x) d x \int_{S} f(y) \varphi_{x} g(y) d y \\
=\int_{S}(f * g)(x) \beta(x) d x=\int_{S} T(f * g) b d m .
\end{aligned}
$$

This holds for all $b \in L^{\infty}(S)$, hence $f * T g=T(f * g)$. The commutativity of $L^{1}(S ; p)$ finishes the proof. Q.E.D.

THEOREM 10b. Suppose $T$ is a bounded multiplier transformation of $L^{1}(S ; p)$ into $L^{1}(S ; p)$, then $T=T_{\mu}$ for some $\mu \in M(S ; p)$. 
Proof. For each $n$, let $(T f)_{n}=t_{n} \hat{f}_{n}$, for all $f \in L^{1}(S ; p)$. Since $[-1,1]$ is separable, there is a countable approximate identity (see $4 \mathrm{~b})\left\{u^{(j)}: j=1,2, \ldots\right\}$, such that for any $f \in L^{1}(S ; p), f * u^{(j)} \rightarrow f\left(L^{1}\right)$ as $j \rightarrow \infty$, and $\left\|u^{(j)}\right\|_{1}=1$. The set $\left\{T u^{(j)}: j=1,2, \ldots\right\}$ is bounded in the $L^{1}$-norm by $\|T\|$, and hence some subsequence of it converges weak-* (in the dual of $C(S ; p)$ ) to a measure $\mu \in M(S ; p)$ with $\|\mu\| \leqq\|T\|$. By dropping some terms and reindexing we may assume $T u^{(j)} \rightarrow \mu$ (weak-*). That is, for each $f \in C(S ; p), \int_{S} f T u^{(j)} d m \rightarrow \int_{S} f d \mu$ as $j \rightarrow \infty$. In particular let $f=\psi_{p}^{-1} P_{n}^{\lambda}$, and then $\left(T u^{(j)}\right)_{n} \rightarrow \hat{\mu}_{n}$, i.e., $t_{n}\left(u^{(j)}\right)_{n}^{\hat{n}} \rightarrow \hat{\mu}_{n}$ as $j \rightarrow \infty$. Since $\left\{u^{(j)}\right\}$ is an approximate identity $\left(u^{(j)}\right)_{n} \rightarrow 1$ as $j \rightarrow \infty$ for each $n$. Hence $t_{n}=\hat{\mu}_{n}$, and $\|\mu\| \leqq\|T\|$. Q.E.D.

THEOREM 10c. There is a Banach algebra isomorphism between $M(S ; p)$ and $B_{G}\left(L^{1}(S)\right)$, given by $\mu \rightarrow T_{\mu}$. Thus each element of $B_{G}\left(L^{1}(S)\right)$ is a multiplier transformation.

Proof. Let $T \in B_{G}\left(L^{1}(S)\right)$. By $10 \mathrm{a}$ and $10 \mathrm{~b}$ there is a $\mu \in M(S ; p)$ such that $T f=f * \mu$ for all $f \in L^{1}(S ; p)$, and $\|\mu\| \leqq\|T\|$. Let $\left\{u_{v}\right\}$ be an approximate identity, $f \in L^{1}(S)$, then $T\left(f * u_{v}\right) \rightarrow T f\left(L^{1}\right)$ since $T$ is bounded. By the relation proved in 10a $T\left(f * u_{v}\right)=f * T u_{v}=f *\left(u_{v} * \mu\right)=\left(f * u_{v}\right) * \mu \rightarrow f * \mu$ as $V$ contracts to 1 . Hence $T f=f * \mu$ for all $f \in L^{1}(S)$. The remaining assertions of the theorem are proved as in 8. Q.E.D.

11. Rotation-commuting projections. The elements of $B_{G}(C(S)), B_{G}\left(L^{1}(S)\right)$, and those of $B_{G}\left(L^{\infty}(S)\right)$ which are multiplier transformations, which are projections, are the idempotents $\left(T^{2}=T\right)$ and correspond to idempotent zonal measures. By Theorem 5d these must satisfy $\hat{\mu}_{N+1}=\hat{\mu}_{N+3}=\cdots$ and $\hat{\mu}_{N}=\hat{\mu}_{N+2}=\cdots$ for some $N$, and $\hat{\mu}_{n}=0$ or 1 for all $n$, and all such possibilities arise.

This also characterizes the rotation-invariant subspaces which are ranges of bounded projections, since by a theorem of Rudin [11], if $Y$ is the image of a bounded projection and $Y$ is invariant under $G$, then $Y$ is the image of a bounded projection which commutes with all elements of $G$.

\section{REFERENCES}

1. S. Bochner, Positive zonal functions on spheres, Proc. Nat. Acad. Sci. U.S.A. 40 (1954), 1141-1147.

2. E. Cartan, Sur la determination d'un système orthogonal complet dans un espace de Riemann symmetrique clos, Rend. Circ. Mat. Palermo 53 (1929), 217-252.

3. C. F. Dunkl, Harmonic analysis on spheres, Ph.D. Thesis, University of Wisconsin, Madison, 1965.

4. A. Erdélyi, et al., Higher transcendental functions (Bateman Manuscript Project), Vols. I, II, McGraw-Hill, New York, 1953.

5. I. M. Gel'fand, Spherical functions on symmetric spaces, Dokl. Akad. Nauk. SSSR 70 (1950), 5-8. 
6. S. Helgason, Differential geometry and symmetric spaces, Academic Press, New York, 1962.

7. H. Helson, Isomorphisms of abelian group algebras, Ark. Mat. 2 (1953), 475-487.

8. I. I. Hirschman, Jr., Harmonic analysis and ultraspherical polynomials, Proceedings of the Conference on Harmonic Analysis, Cornell, 1956.

9. L. Koschmieder, Über die C-Summierbarkeit gewisser Reihen von Didon und Appell, Math. Ann. 104 (1931), 387-402.

10. W. Rudin, Fourier analysis on groups, Interscience, New York, 1962.

11. - Projections in invariant subspaces, Proc. Amer. Math. Soc. 13 (1962), 429-432.

Princeton University,

Princeton, New Jersey 